\title{
A PET microdosing study with the P-glycoprotein inhibitor tariquidar
}

\author{
Martin Bauer ${ }^{1}$, Markus Zeitlinger ${ }^{1}$, Cécile Philippe ${ }^{2}$, Johann Stanek ${ }^{1,3}$, Wolfgang Wadsak², Markus Mitterhauser ${ }^{2}$, \\ Georgios Karanikas ${ }^{2}$, Markus Müller ${ }^{1}$, Oliver Langer ${ }^{1,3^{*}}$ \\ From 18th Scientific Symposium of the Austrian Pharmacological Society (APHAR). Joint meeting with the \\ Croatian, Serbian and Slovenian Pharmacological Societies. \\ Graz, Austria. 20-21 September 2012
}

\section{Background}

The adenosine triphosphate-binding cassette transporters P-glycoprotein (Pgp) and breast cancer resistance protein (BCRP) restrict absorption and body distribution and promote excretion of several clinically used drugs. Tariquidar (XR9576) is a potent third-generation dual Pgp and BCRP inhibitor, which is currently tested in clinical trials to overcome chemoresistance of tumors and to enhance brain distribution of Pgp/BCRP substrate drugs. We performed a positron emission tomography (PET) microdosing study with carbon-11-labelled tariquidar $\left(\left[{ }^{11} \mathrm{C}\right]\right.$ tariquidar $)$ which aimed at assessing the brain distribution of $\left[{ }^{11} \mathrm{C}\right]$ tariquidar in healthy volunteers.

\section{Methods}

Six healthy subjects received an i.v. bolus injection of approximately $400 \mathrm{MBq}$ of $\left[{ }^{11} \mathrm{C}\right]$ tariquidar containing less than $30 \mu \mathrm{g}$ of unlabelled tariquidar. Then, dynamic brain PET scans and arterial blood sampling were performed. Radiolabelled metabolites of $\left[{ }^{11} \mathrm{C}\right]$ tariquidar in plasma were measured with a solid-phase extraction/HPLC assay. Brain activity uptake was expressed as the ratio of the area under the whole brain grey matter time-activity curve to the area under the plasma time-activity curve from time 0 to 60 min $\left(\mathrm{AUC}_{0-60 \text { brain }} / \mathrm{AUC}_{0-60 \text { plasma }}\right)$.

\section{Results}

Brain activity uptake was low after injection of $\left[{ }^{11} \mathrm{C}\right]$ tariquidar with a mean $\mathrm{AUC}_{0-60}$ brain $/ \mathrm{AUC}_{0-60}$ plasma of $0.14 \pm 0.03$. At $60 \mathrm{~min}$ after radiotracer injection, $78 \pm$ $12 \%$ of total radioactivity in plasma was in the form of unchanged parent radiotracer. Less than $1 \%$ of the total injected dose excreted in urine over $90 \mathrm{~min}$.

\section{Conclusions}

Low brain uptake of radioactivity is consistent with tariquidar being, at microdoses, a dual substrate of Pgp and BCRP. $\left[{ }^{11} \mathrm{C}\right]$ Tariquidar PET after inhibition of Pgp with unlabelled tariquidar may be a promising approach to selectively assess BCRP function at the human blood-brain barrier.

\section{Acknowledgements}

Funded by the European Community's Seventh Framework Program (grant agreement 201380 (Euripides)) and Austrian Science Fund (FWF) project "Transmembrane Transporters in Health and Disease" (SFB F35).

\section{Author details}

'Department of Clinical Pharmacology, Medical University of Vienna, 1090 Vienna, Austria. ${ }^{2}$ Department of Nuclear Medicine, Medical University of Vienna, 1090 Vienna, Austria. ${ }^{3}$ Health and Environment Department, AIT Austrian Institute of Technology GmbH, 2444 Seibersdorf, Austria.

Published: 17 September 2012

doi:10.1186/2050-6511-13-S1-A17

Cite this article as: Bauer et al:: A PET microdosing study with the Pglycoprotein inhibitor tariquidar. BMC Pharmacology and Toxicology 2012 13(Suppl 1):A17.

\footnotetext{
* Correspondence: oliver.langer@meduniwien.ac.at

${ }^{1}$ Department of Clinical Pharmacology, Medical University of Vienna, 1090

Vienna, Austria

Full list of author information is available at the end of the article
}

(c) 2012 Bauer et al; licensee BioMed Central Ltd. This is an Open Access article distributed under the terms of the Creative Commons 